Draft Version November 23, 2018

Preprint typeset using $\mathrm{LAT}_{\mathrm{E}} \mathrm{X}$ style emulateapj v. 11/10/09

\title{
LOWER LIMITS ON ULTRAHIGH-ENERGY COSMIC RAY AND JET POWERS OF TEV BLAZARS
}

\author{
Soebur Razzaque ${ }^{1,2}$, Charles D. Dermer ${ }^{3}$, Justin D. Finke ${ }^{3}$ \\ Draft version November 23, 2018
}

\begin{abstract}
Lower limits on the power emitted in ultrahigh-energy cosmic rays (UHECRs), which are assumed to be protons with energy $\gtrsim 10^{17}-10^{20} \mathrm{eV}$, are derived for $\mathrm{TeV}$ blazars with the assumption that the observed $\mathrm{TeV} \gamma$ rays are generated due to interactions of these protons with cosmic microwave photons. The limits depend on the spectrum of the injected UHECR protons. While for a -2.2 injection spectrum, the lower limits on the powers emitted in UHECRs by 1ES 0229+200, 1ES 1101232 and 1ES 0347-121 are lower than their respective synchrotron luminosities $\left(\sim 10^{46} \mathrm{erg} \mathrm{s}^{-1}\right)$; in the case of 1ES 1426+428 it exceeds the corresponding synchrotron luminosity by up to an order of magnitude. The proposed Auger North Observatory should be able to detect $4 \times 10^{19} \mathrm{eV}$ cosmic ray protons from $1 \mathrm{ES} 1426+428$ within a few years of operation and test the $\mathrm{TeV} \gamma$-ray production model by UHECR energy losses while propagating along the line-of-sight, or constrain the intergalactic magnetic field to be larger than $\sim 10^{-16} \mathrm{G}$ in case of no detection. The lower limits on the apparentisotropic jet power from accelerated $10^{10}-10^{20} \mathrm{eV}$ proton spectra in the blazar jet is of the order of the Eddington luminosity of a $10^{9} M_{\odot}$ black hole for a cosmic-ray injection spectrum -2.2 or harder for all blazars considered except for 1ES 1426+428. In the case of the latter the apparent-isotropic jet power exceeds the Eddington luminosity by an order of magnitude. For an injection spectrum softer than -2.2 , as is required to fit the observed cosmic-ray data above $\sim 10^{17}-10^{18} \mathrm{eV}$, the Eddington luminosity is exceeded by the lower limits on the jet power for all blazars considered.

Subject headings: cosmic rays - galaxies:active - gamma rays: galaxies - gamma rays: general gamma rays: ISM
\end{abstract}

\section{INTRODUCTION}

The origin and composition of the highest-energy cosmic rays (CRs) are still unknown (see, e.g., Gaisser 2010). They are generally thought to be produced in extragalactic sources, due to a lack of powerful Galactic sources capable of producing them and due to their near-isotropic arrival direction distribution on large scales (Abraham et al. 2009). The Galactic supernova remnants are leading candidates for producing CRs below $\sim 10^{17} \mathrm{eV}$ (see, e.g., Ptuskin et al. 2010). Above this energy, denoted as ultrahigh energy (UHE), observed CRs may originate in active galactic nuclei (AGNs) with blazars being favored (Rachen \& Biermann 1993; Berezinsky et al. 2006; Dermer et al. 2009; Pe'er et al. 2009) and/or in gamma-ray bursts (GRBs; Waxman 2005; Vietri 1995; Wick et al. 2004; Razzaque et al.|2010) along with their low-luminosity counterparts (Murase et al. 2006; Wang et al. 2007).

In the context of the AGN/blazar hypothesis, lack of a significant correlation between the known position of the sources and the arrival directions of the UHECRs (Abraham et al. 2009) is generally explained by deflections of the charged primaries in the Galactic and intergalactic magnetic field (IGMF). The value of the IGMF is not known but limits exist, $\lesssim 10^{-6} \mathrm{G}$ from

\footnotetext{
1 School of Physics, Astronomy and Computational Sciences, George Mason University, Fairfax, Virginia 22030; srazzaqu@gmu.edu

2 Present address: Space Science Division, U.S. Naval Research Laboratory, Washington, DC 20375

3 Space Science Division, U.S. Naval Research Laboratory, Washington, DC 20375
}

cosmological observations (see, e.g., Barrow et al. 1997; Kahniashvili et al. 2009), and $\gtrsim 10^{-15}-10^{-16} \mathrm{G}$ assuming TeV blazars are persistent sources Neronov \& Vovk 2010; Tavecchio et al. 2010b) while $>10^{-17}-10^{-18} \mathrm{G}$ assuming TeV blazars are variable (Dolag et al. 2011; Dermer et al. 2011; Taylor et al. 2011). UHECRs propagating in voids, with very small value of the IGMF, may avoid significant deflection and reach us directly from their sources, if nearby. For sources beyond $\sim$ $100 \mathrm{Mpc}$, the highest-energy primary CRs cannot reach us since they lose energy by interacting with the lowenergy photons of the Cosmic Microwave Background (CMB) and the Extragalactic Background Light (EBL), a phenomenon known as the GZK effect (Greisen 1966; Zatsepin \& Kuz'min 1966). The secondary particles however, can form an electromagnetic cascade and cascade radiation, mostly Compton-scattered CMB photons (see, e.g., Dai et al. 2002; Razzaque et al. 2004; Ichiki et al. 2008; Tavecchio et al. 2010b; Dermer et al. 2011), can reach us as if emitted from the source, if the IGMF value is sufficiently small.

Indeed it has been proposed recently that $\mathrm{TeV} \gamma$-ray emission detetcted from distant blazars and showing no significant flux variation is secondary cascade emission by UHECR protons (Essey \& Kusenko 2010; Essey et al. 2010, 2011). This proposition opens a possibility to estimate the UHECR power and subsequently the jet power of the TeV blazars, since protons are expected to dominate the energy budget in such a scenario. In an alternate scenario, non-variation of $\mathrm{TeV}$ flux may arise from cascade emission in EBL and CMB from $\gtrsim 10 \mathrm{TeV}$ photons, which could be distinguished from proton-induced cascade emission at very high energies (Murase et al. 
2011). These models, however, cannot explain rapid variability, often on hours time scale and over broad frequency range, observed in the bulk population of blazars and thought to be related to the size scale of the emission region in the jet. The broadband radio to $\gamma$ ray spectral energy distribution of $\mathrm{TeV}$ blazars consists of two peaks: A low-energy peak typically in the UV/Xray and a high-energy peak at $\sim 100 \mathrm{GeV}$ (see, e.g., Giommi \& Padovani 1994; Aharonian et al. 2008; Tavecchio et al. 2010a). While the low-energy peak is generally modeled as synchrotron radiation, the highenergy peak is often modeled as inverse Compton (IC) emission by the sychrotron-radiating electrons (see, e.g., Costamante \& Ghisellini 2002; Böttcher 2007) or by hadronic emission (Mücke et al. 2003). Apart from apparent non-variation of the flux at $\gtrsim \mathrm{TeV}$ in a number of high-redshift blazars, models of high-energy $\gamma$ ray emission from the jet often requires very hard intrinsic spectrum in order to avoid rapid flux reduction via $\gamma \gamma$ pair production with EBL photons (see, e.g., Franceschini et al. 2008; Finke et al. 2010). Emission from UHECR cascade in the EBL-CMB can potentially evade these issues (Essey et al. 2010, 2011).

In this paper we derive lower limits on the UHECR and jet powers of the $\mathrm{TeV}$ blazars by assuming that the total energy loss rate, integrated over energies $\gtrsim 10^{17} \mathrm{eV}$, by UHECR protons in the electromagnetic channels $\left(p \gamma_{\mathrm{CMB}} \rightarrow e^{+} e^{-} ; p \gamma_{\mathrm{CMB}} \rightarrow \pi^{0} \rightarrow \gamma \gamma, \rightarrow \pi^{ \pm} \rightarrow e^{ \pm}\right)$and after propagating to a redshift $z_{\gamma}$, when the universe becomes transparent to $\mathrm{TeV} \gamma$ rays, is equal to the bolometric $\mathrm{TeV} \gamma$-ray luminosity calculated from the observed spectra and $z_{\gamma}$. For the EBL models, not constrained by the Fermi Large Area Telescope (LAT) data (Abdo et al. 2010c), the $\gamma \gamma$ opacity $\tau_{\gamma \gamma} \approx 1$ at $z_{\gamma} \approx 0.1$. For $z<z_{\gamma}$ cascade emission is not reprocessed efficiently and is mostly emitted at very-high energies, far above the $\sim 0.1 \mathrm{TeV}$ threshold of the air Cherenkov telescopes but could potentially be detected by the proposed HiSCORE $\gamma$-ray detector (Hampf et al. 2011) which would be sensitive in the $\mathrm{PeV}$ rage.

Our limits are based on the assumption that UHECR protons do not suffer significant energy losses in the blazar jet and escape along the direction within the jet opening angle. As mentioned earlier, $\mathrm{TeV} \gamma$ rays that are produced in the jet from UHECR interactions, suffer attenuation in the EBL. Because of this reduction of the $\gamma$-ray source flux, the total required UHECR power is greater if the observed flux is a combination of the source and cascade fluxes than if solely coming from the cascade. We do not include deflections of the primary proton and cascade $e^{+} e^{-}$in the IGMF, which reduce the flux of the secondary radiation and increase the required UHECR and jet powers (see, e.g., Razzaque et al. 2004; Dermer et al. 2011). Moreover, large $\gtrsim 10^{-9} \mathrm{G}$ magnetic field is expected in the filaments in the galaxy clusters (Rvu et al. 2008) which can also reduce the cascade radiation flux when encountered by the primary proton and cascade $e^{+} e^{-}$, thus further increasing the required UHECR and jet powers. Our limits, though model-dependent, are conservative in this sense. They will be weaker if other processes, e.g. leptonic emission from the jet and/or from the cascade in EBL-CMB, contribute significantly to the observed $\mathrm{TeV}$ data. Based on our estimated lower limits on the UHECR fluxes from the $\mathrm{TeV} \gamma$-ray blazars, we also calculate the detection rate of the primary UHECR protons, which travel along the line-of-sight from their sources, by the Pierre Auger Observatory

We calculate the energy losses by UHECR protons in the CMB in Sec. 2, and estimate the apparent-isotropic luminosity of $\mathrm{TeV}$ blazars from spectra measured by air Cherenkov telescopes in Sec. 3. We derive our limits on the UHECR and jet powers in Sec. 4, and calculate UHECR event rate based on the limits in Sec. 5. We discuss results and conclude our investigation in Sec. 6.

\section{COSMIC RAY SPECTRA AND ENERGY LOSSES}

We calculate the power-law source spectrum (total number of particles generated isotropically from the source per unit energy and time intervals) with an index $-\kappa$ and between the generation energies $E_{1 g}-E_{2 g}$, given an apparent-isotropic bolometric luminosity (power) of cosmic rays $L_{\mathrm{CR}}$, as

$$
q\left(E_{g}\right) \equiv \frac{d N}{d E_{g} d t}=\frac{L_{\mathrm{CR}}(\kappa-2) E_{1 g}^{\kappa-2}}{1-\left(E_{1 g} / E_{2 g}\right)^{\kappa-2}} E_{g}^{-\kappa} ; \kappa \neq 2 .
$$

The observed energy of a CR on the Earth, however, is degraded due to adiabatic or redshift (due to expansion of the universe) loss and due to collisional losses with the CMB and/or EBL photons. The relationship between the generated $\mathrm{CR}$ energy $E_{q}(z)$ at redshift $z$ and the observed energy $E$ is (Berezinsky et al. 2006)

$$
\begin{aligned}
E= & E_{g}(z)-\int_{0}^{z} d z^{\prime} \frac{E_{g}\left(z^{\prime}\right)}{1+z^{\prime}} \\
& -\int_{0}^{z} d z^{\prime} \frac{1+z^{\prime}}{H\left(z^{\prime}\right)} b_{0}\left[\left(1+z^{\prime}\right) E_{g}\left(z^{\prime}\right)\right],
\end{aligned}
$$

where $E_{g}\left(z^{\prime}\right)=E_{g}(z)\left(1+z^{\prime}\right) /(1+z), H\left(z^{\prime}\right)$ is the comoving Hubble parameter and $b_{0}(E)=-d E / d t$ is the energy loss rate of an UHECR at the present epoch $(z=0)$. UHECR protons lose their energy by interacting dominantly with the CMB photons, creating $e^{+} e^{-}$ pairs and $\pi$ 's. These energy-loss rates and their evolution with redshift have been calculated by many authors, and they are well understood in the $10^{17}-10^{20} \mathrm{eV}$ energy range (see, e.g., Stanev et al. 2000; Berezinsky et al. 2006; Dermer \& Menon 2009).

The flux of cosmic rays on the Earth from a source at redshift $z$ is then

$$
\begin{aligned}
J(E) & =\frac{q\left(E_{g}\right)}{4 \pi d_{c}^{2}(1+z)} \frac{d E_{g}}{d E} \\
& \approx \frac{L_{\mathrm{CR}}(\kappa-2) E_{1}^{\kappa-2}}{4 \pi d_{c}^{2}(1+z)^{3-\kappa}} E^{-\kappa}\left(\frac{E_{g}}{E}\right)^{-\kappa} \frac{d E_{g}}{d E} ; \kappa \neq 2,(3)
\end{aligned}
$$

where the second line follows with an approximation $E_{1 g} \ll E_{2 g}$ in Equation (11) and when the lowest-energy CRs lose energy due to redshift only, i.e. $E_{1 g}=E_{1}(1+z)$. The Jacobian factor $d E_{a} / d E$ follows from Equation (2) and is given by Berezinsky et al. (2006). The comoving distance $d_{c}$, which is related to the proper distance $d_{p}$ and the luminosity distance $d_{L}$ as $d_{L}=(1+z) d_{c}=(1+z)^{2} d_{p}$,

\footnotetext{
4 http://www .auger.org/
} 


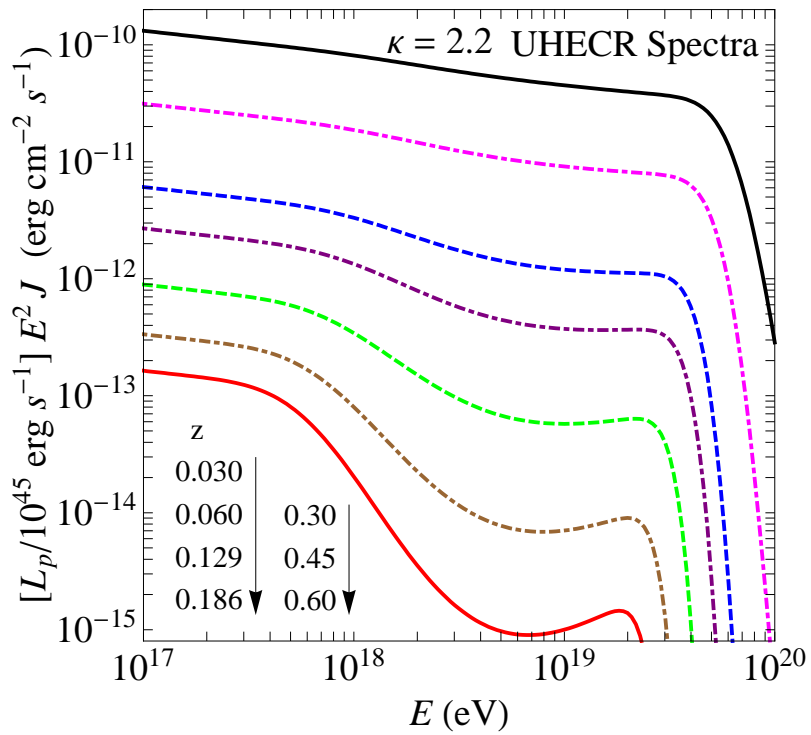

FIG. 1. - Expected UHECR proton spectra on the Earth from a source with increasing redshift from the top to bottom according to Equation (3). We assumed an apparent-isotropic UHECR source luminosity to be $10^{45} \mathrm{erg} \mathrm{s}^{-1}$ with a power-law spectrum of index $\kappa=2.2$ in the generated energy range $10^{17}-10^{20} \mathrm{eV}$. Adiabatic (redshift), $e^{+} e^{-}$pair and $\pi$ creation losses have been taken into account in the numerical calculation.

is defined as (see, e.g., Hogg 1999)

$$
d_{c}=\frac{c}{H_{0}} \int_{0}^{z} \frac{d z^{\prime}}{\sqrt{\Omega_{m}\left(1+z^{\prime}\right)^{3}+\Omega_{\Lambda}}} .
$$

We assume the standard cosmological parameters: $\Omega_{m}=$ $0.27, \Omega_{\Lambda}=0.73$ and a Hubble constant $H_{0}=$ $71 \mathrm{~km} \mathrm{~s}^{-1} \mathrm{Mpc}^{-1}$.

Figure 1 shows the UHECR proton spectra as would be observed from a source at various redshifts but with the same apparent-isotropic luminosity $L_{p}=10^{45} \mathrm{erg} \mathrm{s}^{-1}$ for $10^{17} \mathrm{eV} \leq E_{g} \leq 10^{20} \mathrm{eV}$ and for the spectral index of the generated protons to be $\kappa=2.2$. Note that the sharp cutoff at the highest energies $\gtrsim 4 \times 10^{19} \mathrm{eV}$ is due to $\pi$ losses (the GZK effect) and the dip below $4 \times 10^{19} \mathrm{eV}$ but above $\sim 10^{18} \mathrm{eV}$ is due to $e^{+} e^{-}$pair losses (Berezinsky et al. 2005). These spectral features shift to lower energy for high-redshift sources. Adiabatic losses dominate at the lowest energy in this plot.

The total bolometric power lost, due to creating $e^{+} e^{-}$ pairs and $\pi$ 's, by the UHECR protons, generated at a redshift $z$ and measured at another redshift $z_{0}$, can be calculated from Equation (11) and Equation (2) considering only collisional losses and by integrating over the generation energy in the range $E_{1 g}-E_{2 g}$. The total power lost in electromagnetic (EM) channels is smaller when considering energy losses to secondary charged particles and photons only, which can initiate electromagnetic cascades by interacting with the CMB and EBL photons. Below $E_{g} \sim 4 \times 10^{19} \mathrm{eV}$, energy losses by UHECRs are mostly in the EM channels. Above $E_{g} \sim 4 \times 10^{19} \mathrm{eV}$, roughly $1 / 3$ of the bolometric power lost, with $\sim 50 \%$ inelasticity, goes into producing neutrinos. Subtracting the neutrino losses we calculate the ratio of the power lost in EM channels, due to propagation, to the generated
UHECR power $L_{\mathrm{CR}}$ as

$$
\begin{aligned}
f_{\mathrm{CR}}= & \frac{(\kappa-2) E_{1 g}^{\kappa-2}}{1-\left(E_{1 g} / E_{2 g}\right)^{\kappa-2}} \frac{c}{H_{0}} \frac{\left(1+z_{0}\right)^{2}}{(1+z)^{2}} \int_{E_{1 g}}^{E_{2 g}} d E_{g} E_{g}^{-\kappa} \\
& \times \int_{z_{0}=z_{\gamma}}^{z} d z^{\prime} \frac{\left(1+z^{\prime}\right) b_{0, \mathrm{EM}}\left[\left(1+z^{\prime}\right) E_{g}\left(z^{\prime}\right)\right]}{\sqrt{\Omega_{m}\left(1+z^{\prime}\right)^{3}+\Omega_{\Lambda}}}
\end{aligned}
$$

Here the lower limit of the redshift integration corresponds to $z_{\gamma}$ at which $\tau_{\gamma \gamma} \approx 1$, since efficient reprocessing of the EM energy losses by UHECRs to observed TeV $\gamma$ rays can take place only above this redshift.

Figure 2 shows the fraction $f_{\mathrm{CR}}$ as a function of the redshift and for different values of the spectral index $\kappa$. The top and bottom panels correspond to the $10^{17}$ $4 \times 10^{19} \mathrm{eV}$ and $10^{17}-10^{20} \mathrm{eV}$ energy ranges of the generated spectra, respectively. The dotteded curves in each panel correspond to the present redshift $\left(z_{0}=0\right)$ in Equation (5). The solid curves correspond to the redshift $z_{0}=z_{\gamma}=0.1$ at which $\gamma \gamma \rightarrow e^{+} e^{-}$pair production opacity $\tau_{\gamma \gamma}=1$ at $\mathrm{TeV}$, according to the EBL models by Finke et al. (2010) and by Franceschini et al. (2008), and those photons can propagate to us without much attenuation (Fazio \& Stecker 1970). Figure 3 shows $f_{\mathrm{CR}}$ as in Fig. 2 but above $10^{18} \mathrm{eV}$ for comparison. Note that the loss fraction increases in this case because a smaller part of the UHECR spectrum is considered here, where collisional losses are the most important. As can be seen, the loss fraction crucially depends on the index $\kappa$ of the generated CR spectrum and is smaller for softer spectra.

The Lorentz factor of the $e^{+} e^{-}$pairs created from a $10^{18} \mathrm{eV}$ proton is roughly $\gamma_{e} \approx 10^{9}$. The Compton-scattered CMB photon energy by the pairs is $(4 / 3) \gamma_{e}^{2} \varepsilon_{\mathrm{CMB}} \approx 900(1+z)\left(\gamma_{e} / 10^{9}\right)^{2} \mathrm{TeV}$. Subsequent absorption of these photons in the EBL, creating $e^{+} e^{-}$ pairs, and Compton scattering of CMB photons give rise to an EM cascade. A similar scenario applies for $\pi^{0} \rightarrow \gamma \gamma$ and $\pi^{ \pm} \rightarrow e^{ \pm}$channels. Compton photons of a given energy from the cascade can reach the Earth once their opacity falls below unity. Thus substantial reprocessing of UHECR power lost in EM channels to observed TeV $\gamma$ rays can only take place at $z_{\gamma} \gtrsim 0.1$. We estimate the total EM power loss by UHECRs from $\mathrm{TeV} \gamma$-ray data in the next section.

\section{TEV BLAZARS AND GAMMA RAY LUMINOSITY}

We assume that the $\mathrm{TeV} \gamma$ rays detected from blazars at $z \gtrsim z_{\gamma} \approx 0.1$ are reprocessed cascade emission from UHE protons with energy $10^{17} \leq E_{g} \leq 10^{20} \mathrm{eV}$, that are generated at the blazars. $\mathrm{TeV}$ emission should not be rapidly variable in this case since cascade emission takes place over a large size scale. There can be some variability at an energy much below $1 \mathrm{TeV}$, due to contamination by the source photons. Currently there are four such $\mathrm{TeV}$ blazars with adequate data, namely 1ES 1426+428 (Aharonian et al. 2003), 1ES 0229+200 Aharonian et al. 2007c; Perkins et al. 2010), 1ES 1101-232 (Aharonian et al. 2007a) and 1ES 0347-121 (Aharonian et al. 2007b). These are all BL Lac objects.

Figure 4 shows the very high-energy $\gamma$-ray data collected from these blazars. None of these sources except 


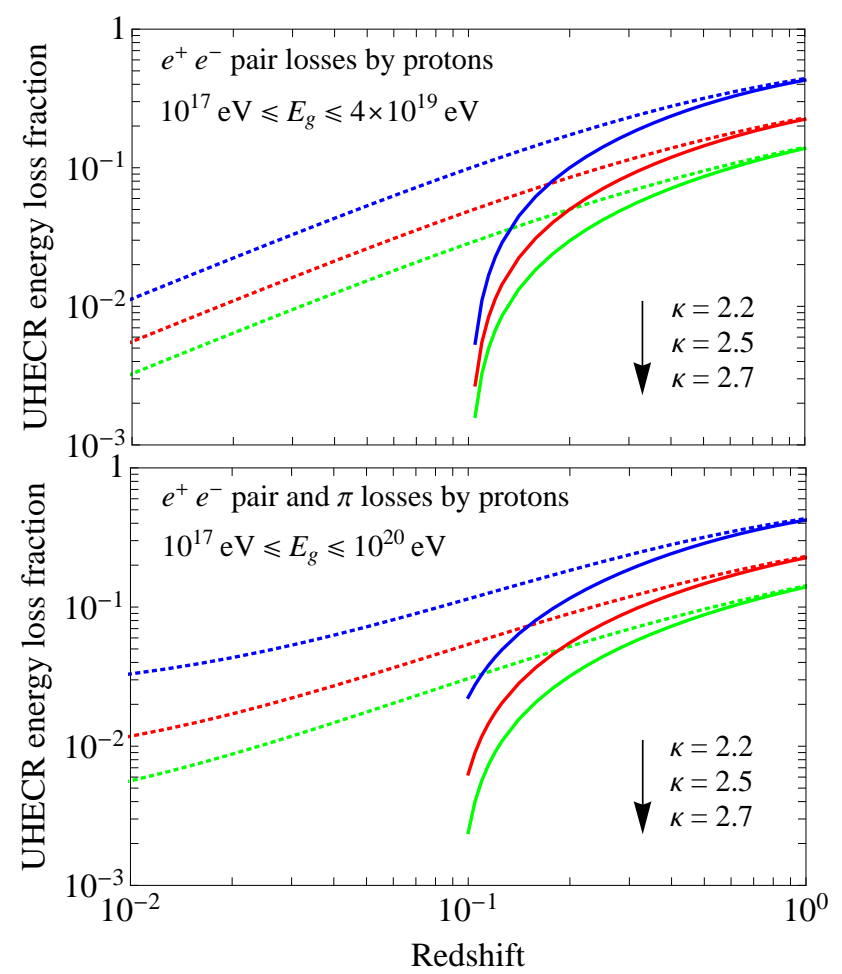

FIG. 2.- Total bolometric power loss fraction by UHECR protons, generated at various redshifts and with different spectral index $\kappa$, propagting to $z=0$ (dotted curves) and to $z=0.1$ (solid curves). The universe becomes optically thin $\left(\tau_{\gamma \gamma} \lesssim 1\right)$ to $\mathrm{TeV}$ photons due to $e^{+} e^{-}$pair creation with the EBL photons at $z \approx 0.1$ (see, e.g., the EBL models by Finke et al. 2010; Franceschini et al. 2008) Top panel - Energy losses by $e^{+} e^{-}$pair creation, which dominates below $\approx 4 \times 10^{19} \mathrm{eV}$, with the CMB photons. Bottom panel - Energy losses by $e^{+} e^{-}$pair and $\pi$ creation, which dominates above $\approx 4 \times 10^{19} \mathrm{eV}$, with the CMB photons. The lowest UHECR energy is assumed to be $10^{17} \mathrm{eV}$.

1ES 1101-232 has been convincingly detected ${ }^{5}$, which is sensitive in the $0.1-300 \mathrm{GeV}$ range. However data at much lower frequencies, Optical-UV and X-ray, are available. Emission at these frequencies is widely believed to be synchrotron radiation from non-thermal electrons in the blazar jet. The bolometric synchrotron luminosity $L_{\text {syn }}$, calculated as ten times the specific luminosity $\nu L_{\nu}$ at the peak of the synchrotron emission as given in Tavecchio et al. (2010a), is also listed in in Table 1 for each of the four blazars.

Figure 4 also shows the deabsorbed $\gamma$-ray data points using the Finke et al. (2010) EBL model at $z_{\gamma}=0.1$. We fit the deabsorbed $\gamma$-ray spectra (black dotted lines) by two smoothly-joined power laws, similar to a Band

TABLE 1

BlazAR AND TeV $\gamma$-RAY MODEL PARAMETERS

\begin{tabular}{ccccc}
\hline \hline Blazar & $z$ & $\begin{array}{c}L_{\mathrm{syn}} \\
(\mathrm{erg} / \mathrm{s})\end{array}$ & $\begin{array}{c}L_{z_{\gamma}=0.1} \\
(\mathrm{erg} / \mathrm{s})\end{array}$ & $\beta$ \\
\hline 1ES 1426+428 & 0.129 & $6 \times 10^{45}$ & $2.1 \times 10^{45}$ & -2.3 \\
1ES 0229+ 200 & 0.139 & $2 \times 10^{46}$ & $4.6 \times 10^{44}$ & -1.7 \\
1ES 1101 - 232 & 0.186 & $3 \times 10^{46}$ & $2.1 \times 10^{44}$ & -2.0 \\
1ES 0347-121 & 0.188 & $2 \times 10^{46}$ & $2.0 \times 10^{44}$ & -2.2 \\
\hline
\end{tabular}

5 A tentative detection of $1 \mathrm{ES} 0229+200$ has been reported by Orr et al. (2011) and by Abdo et al. (2010b)

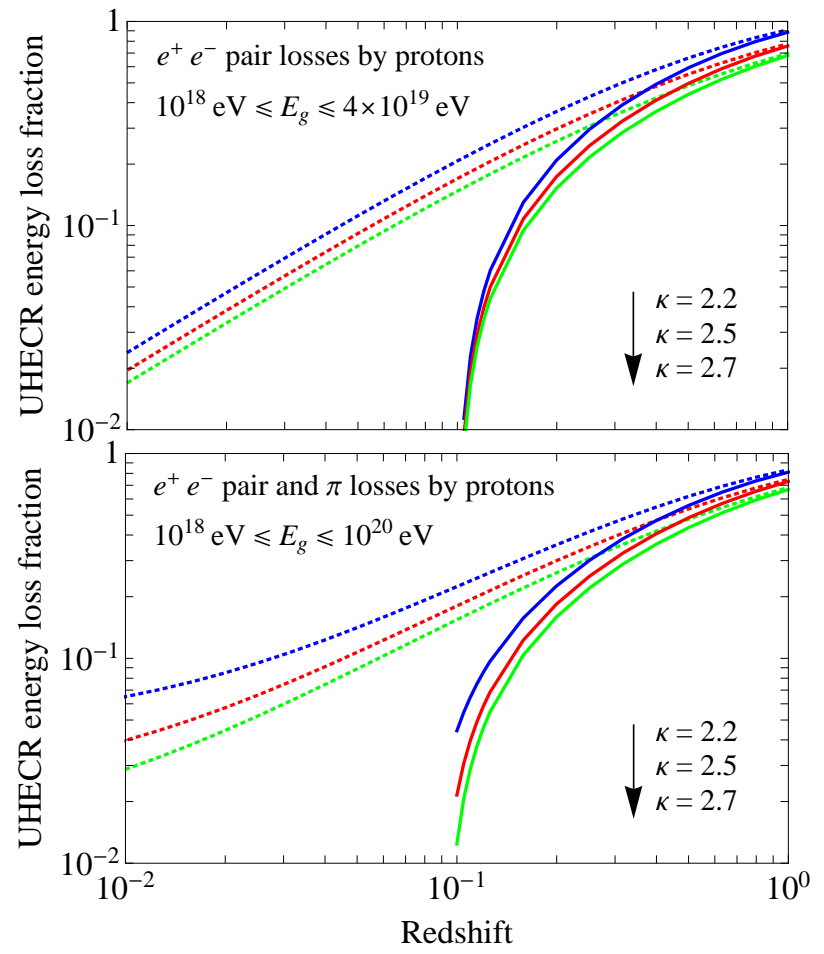

FIG. 3.- The same as in Fig. 2 but the lowest UHECR energy is assumed to be $10^{18} \mathrm{eV}$.

spectrum (Band et al. 1993), with a super-exponential cutoff at the high energies as below

$$
\frac{d N}{d \varepsilon d A d t}=C\left\{\begin{array}{l}
\varepsilon_{\mathrm{GeV}}^{\alpha} \exp \left[\frac{(2+\alpha) \varepsilon}{\varepsilon_{\mathrm{pk}}}\right] ; \varepsilon<\frac{(\alpha-\beta) \varepsilon_{\mathrm{pk}, \mathrm{GeV}}}{2+\alpha} \\
\varepsilon_{\mathrm{GeV}}^{\beta}\left[\frac{(\alpha-\beta) \varepsilon_{\mathrm{pk}, \mathrm{GeV}}}{2+\alpha}\right]^{\alpha-\beta} \\
\quad \times e^{\beta-\alpha} e^{-\left(\varepsilon / \varepsilon_{c}\right)^{4}} ; \varepsilon \geq \frac{(\alpha-\beta) \varepsilon_{\mathrm{pk}, \mathrm{GeV}}}{2+\alpha}
\end{array}\right.
$$

We keep the index $\alpha$ of the low-energy power law fixed such that $\nu F_{\nu} \propto \nu^{2}(\alpha=0)$, the hardest Compton spectrum expected, to be conservative. The break energy $\varepsilon_{\mathrm{pk}}$, at which the $\nu F_{\nu}$ spectrum turns over, the high-energy power-law index $\beta$ and the cutoff energy $\varepsilon_{c}$ were allowed to vary.

Finally we calculate a conservative lower limit on the apparent-isotropic $\mathrm{TeV} \gamma$-ray luminosity from our fitted spectra (see Fig. 4, dashed lines) to the deabsorbed data points at $z_{\gamma}=0.1$ as

$$
L_{z_{\gamma}=0.1}=4 \pi d_{L}^{2}\left(z_{\gamma}\right) \int d \nu F_{\nu}
$$

in the $10 \mathrm{GeV}-20 \mathrm{TeV}$ range. This luminosity and the high-energy photon index $\beta$ in Equation (6) along with the synchrotron luminosity for individual blazars are listed in Table 1.

\section{LIMITS ON UHECR AND JET POWERS}

We calculate the lower limits on the apparent-isotropic UHECR power of the TeV blazars from $\gamma$-ray luminosity calculated in Equation (7) as

$$
L_{\mathrm{UHECR}}>L_{z_{\gamma}=0.1} / f_{\mathrm{CR}}
$$

Note that for high EBL models (see, e.g., Stecker et al. 2006), with $z_{\gamma}<0.1, L_{z_{\gamma}}$ will be lower. A lower $z_{\gamma}$ 

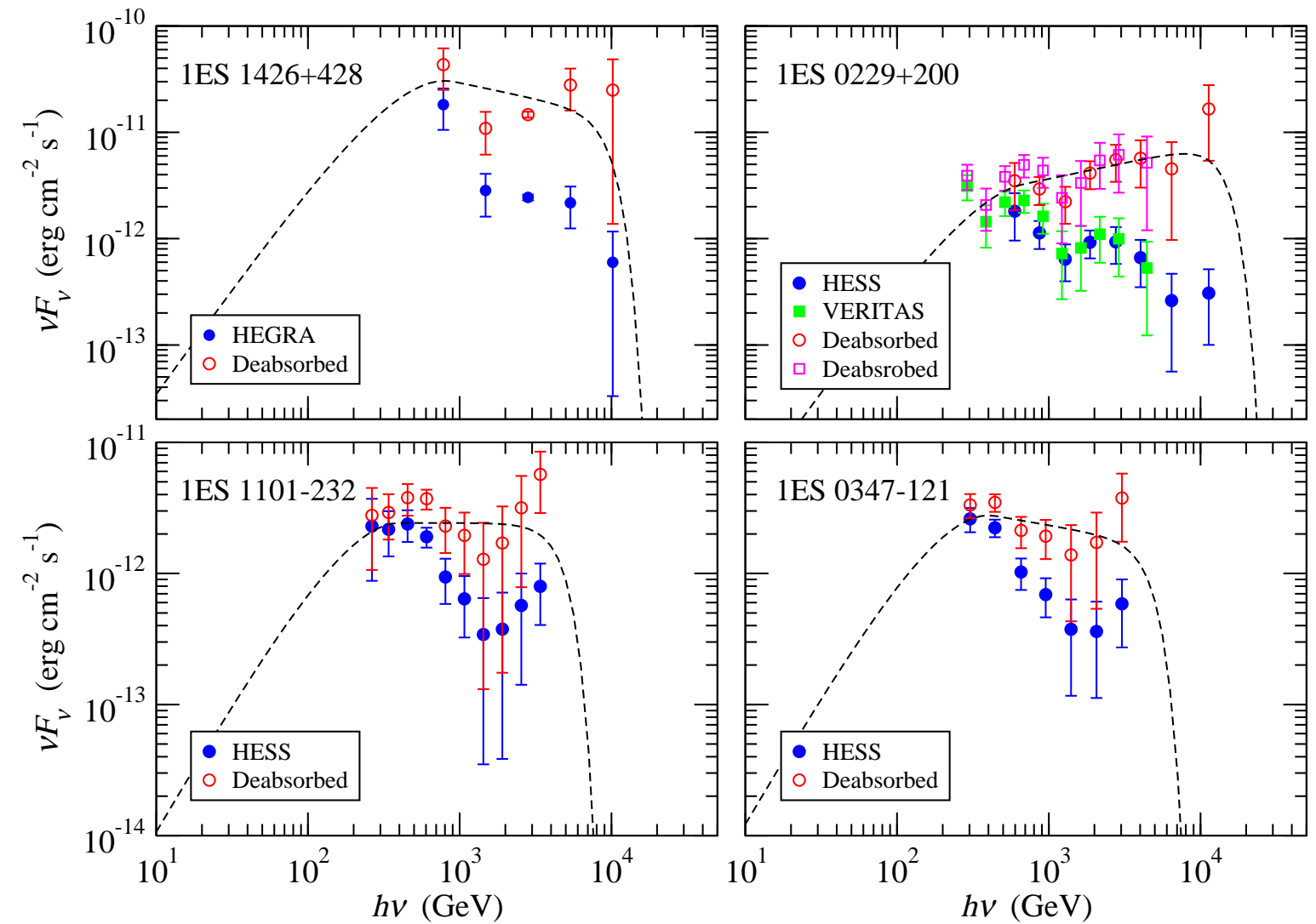

FIG. 4.- TeV $\gamma$-ray data (filled points) from the HESS and VERITAS observations on selected blazars at $z \gtrsim 0.1$. Fits (dashed lines) to the $\gamma$-ray data from these blazars are performed after deabsorption (empty points) at $z=0.1$ with the EBL model by Finke et al. (2010). The fit function consists of two smoothly- joined power laws with a super-exponential, $\exp \left(-\nu^{4}\right)$, cutoff at high energies as in Equation (6) . The low-energy slope of the power law is kept fixed so that $\nu F_{\nu} \propto \nu^{2}$, analogous to the hardest IC spectrum. The high-energy index $\beta$ is listed in Table 1.

also gives a higher $f_{\mathrm{CR}}$ or more efficient conversion of UHECR energy to EM energy. Thus our limits will be weaker for high EBL models, a result consistent with what Essey et al. (2011) found by modeling $\mathrm{TeV} \gamma$-ray data.

The top panel of Fig. 5 shows the lower limits on the apparent-isotropic UHECR power, in the $10^{17}-10^{20} \mathrm{eV}$ range, of individual blazars that we have derived from the $\mathrm{TeV} \gamma$-ray luminosity $L_{z_{\gamma}=0.1}$ listed in Table 1 and Equation (8). The limits are in the range $10^{45}$ $10^{47} \mathrm{erg} / \mathrm{s}$ and are consistent with the required UHECR source luminosity from detailed modeling of $\mathrm{TeV} \gamma$-ray data (Essev et al. 2010).

The bottom panel of Fig. 5 shows the lower limits on the blazar jet power that we have derived from the $\mathrm{TeV}$ $\gamma$-ray data, and are plotted against the synchrotron luminosities of the corresponding blazars. To calculate these lower limits we have extrapolated the proton spectrum down to a minimum energy of $10 \mathrm{GeV}$. Thus the bulk Lorentz factor of the relativistic $\mathrm{TeV}$ blazar jets need to be $\sim 10$, which is consistent with measured values. The empty symbols (as in the top panel) correspond to the limits assuming the same power-law index $\kappa$ as above $10^{17} \mathrm{eV}$ and as is expected in a Fermi shock-acceleration scenario. The filled symbols correspond to the limits assuming $\kappa=2.2$ below $10^{17} \mathrm{eV}$ and $\kappa=2.5$ or 2.7 above $10^{17} \mathrm{eV}$. Such broken powerlaw spectra may arise in more complicated scenarios, e.g. from cooling of UHECR protons in the jet. In case the minimum proton energy is $100 \mathrm{GeV}$, our limits will be weaker by factors $1.6,3.2$ and 5.0 for $\kappa=2.2,2.5$ and 2.7 respectively, in case of single power-law spectrum. Also shown (dotted line) is the Eddington luminosity $L_{\mathrm{Edd}}=1.3 \times 10^{47}\left(M_{\mathrm{bh}} / 10^{9} M_{\odot}\right) \mathrm{erg} / \mathrm{s}$ of a $10^{9} M_{\odot}$ blackhole, which is the typical black-hole mass thought to power the blazar jets.

In case the $\mathrm{TeV}$ blazars produce UHECRs above $10^{18} \mathrm{eV}$ rather than above $10^{17} \mathrm{eV}$, our limits on the UHECR power becomes lower (see Fig. 6 top panel) by a factor of $\sim 2-5$, depending on the injection spectral index $\kappa$. This is an expected result since the blazars need to power a smaller part of the UHECR spectrum in this case. The lower limits on the jet power (Fig. 6 bottom panel) in case of broken power-law spectra (filled symbols) are also reduce by a factor $\sim 2-3$ while shifting the break energy from $10^{17} \mathrm{eV}$ to $10^{18} \mathrm{eV}$, for similar reasons as for the limits on the UHECR power. Our limits on the jet power for single power-law spectra (empty symbols in Fig. 6 bottom panel) from $10^{10} \mathrm{eV}$ to $10^{20} \mathrm{eV}$, however, remains unchanged.

The limits that we have derived are based on the assumption that primary UHECRs and the cascade emission are concentrated within the opening angle of the blazar jet, $\theta_{\text {jet }} \approx 0.1$. For $10^{17} \mathrm{eV} \mathrm{CRs}$ and a characteristic propagation distance of $d_{\mathrm{p}} \approx 500 \mathrm{Mpc}$ with $l_{\text {coc }} \approx 1 \mathrm{Mpc}$ coherence length scale of IGMF, this im- 


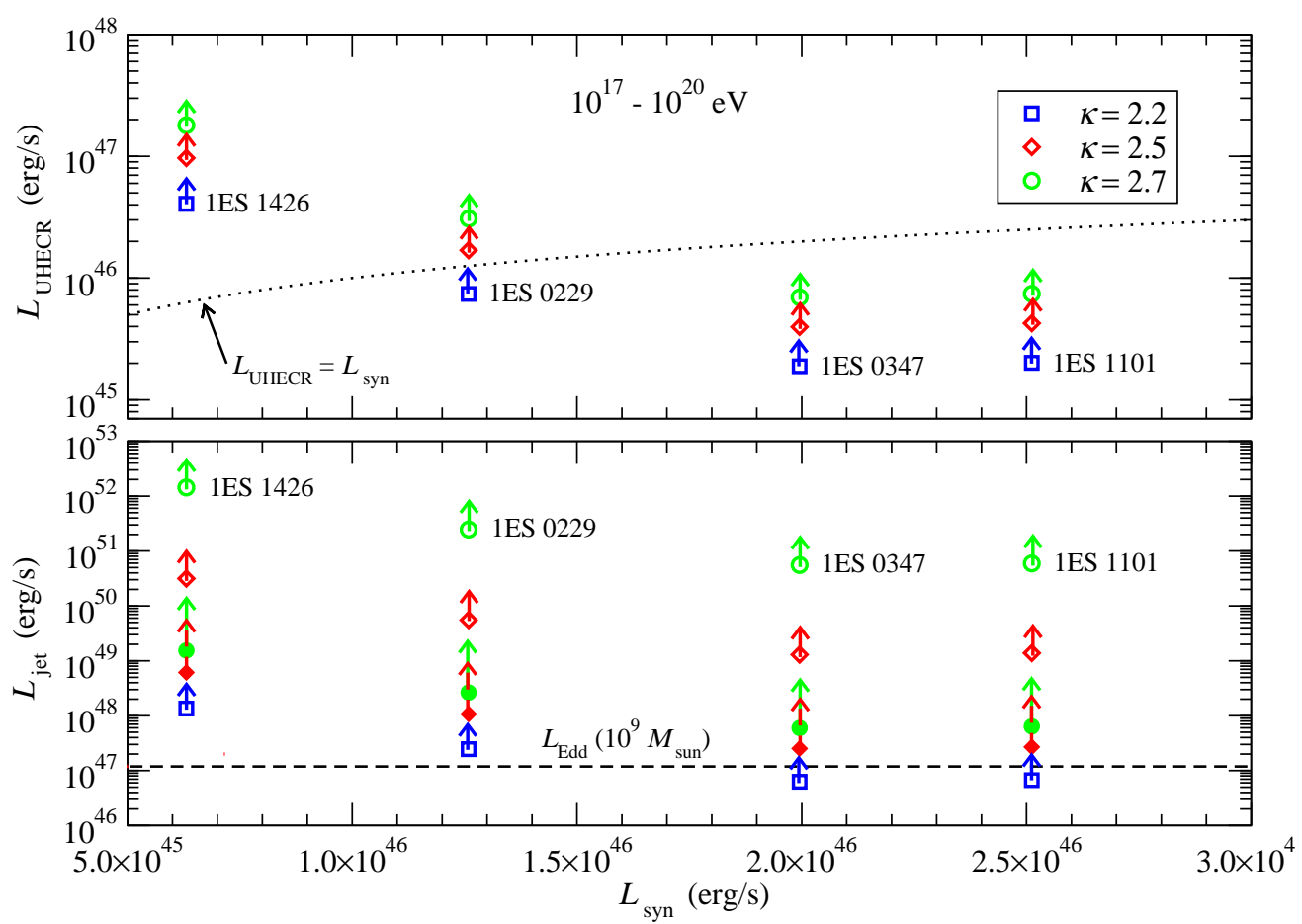

FIG. 5. - Top panel - Lower limits on the apparent-isotropic UHECR power, in the $10^{17}-10^{20} \mathrm{eV}$ range and assuming different injection spectral index $\kappa$, from $\mathrm{TeV}$ blazars derived from the $\mathrm{TeV} \gamma$-ray luminosity $L_{z_{\gamma}=0.1}$ in Table 1 . The limits on the UHECR power are plotted against the synchrotron power (mostly in X rays) of the respective blazars, and the two powers are equal at the dotted line. Bottom panelLower limits on the apparent-isotropic jet power derived from $\mathrm{TeV} \gamma$-ray data and plotted against their respective synchrotron luminosities. The jet powers are calculated from the CR spectra in the $10^{10}-10^{20} \mathrm{eV}$ range by extrapolating the spectrum below $10^{17} \mathrm{eV}$, with single power law index $\kappa$ as above $10^{17} \mathrm{eV}$ (empty symbols), and with broken power law of fixed index $\kappa=2.2$ below $10^{17} \mathrm{eV}$ but varying $\kappa$ above $10^{17} \mathrm{eV}$ (filled symbols). Also shown (dashed line) is the Eddington luminosity of a $10^{9} M_{\odot}$ blackhole.

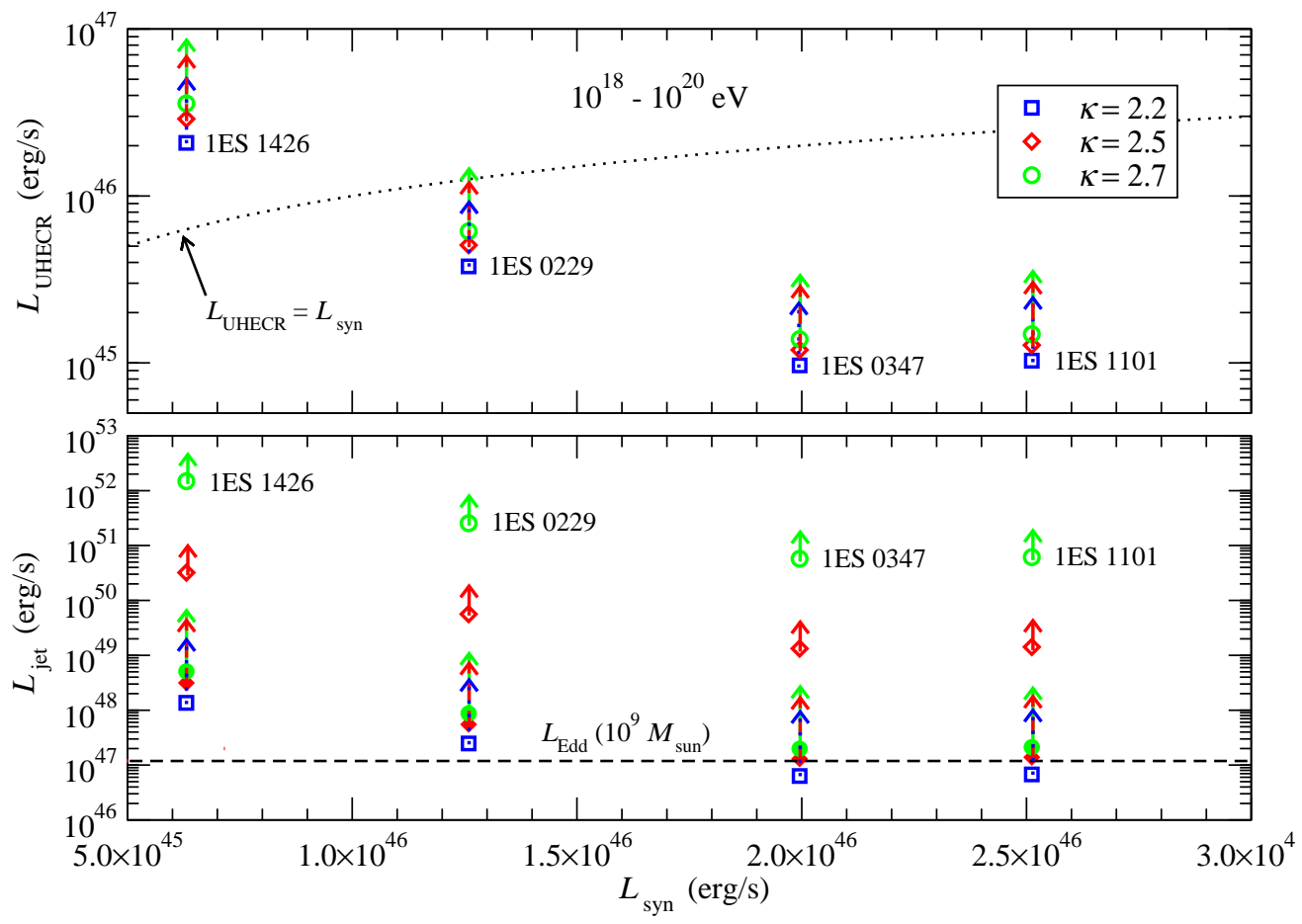

FIG. 6. - The same as in Fig. 5 but assuming that UHECRs are generated in the $10^{18}-10^{20}$ eV range. 
plies that the mean IGMF strength should be

$$
\begin{aligned}
B_{\text {IGMF }, \text { UHECR }} \lesssim & 10^{-12}\left(\frac{\theta_{\text {jet }}}{0.1}\right)\left(\frac{E}{10^{17} \mathrm{eV}}\right) \\
& \times\left(\frac{d_{p}}{500 \mathrm{Mpc}}\right)^{-1 / 2}\left(\frac{l_{\text {coh }}}{\mathrm{Mpc}}\right)^{-1 / 2} \mathrm{G},
\end{aligned}
$$

for the deflection angle to be smaller than $\theta_{\text {jet }}$. A stronger constraint on the IGMF, however, comes from the requirement that the cascade electrons of Lorentz factor $\gamma_{e} \approx 10^{6}$ that upscatter $\mathrm{CMB}$ photons to $\mathrm{TeV} \gamma$ rays do not deviate out of $\theta_{\text {jet }}$. The cooling length scale of electrons in nearby universe is $\lambda_{\mathrm{IC}}=0.71\left(\gamma_{e} / 10^{6}\right)^{-1} \mathrm{Mpc}$, and to avoid any deflection larger than $\theta_{\text {jet }}$ one requires

$$
B_{\mathrm{IGMF}, \mathrm{TeV}} \lesssim 10^{-16}\left(\frac{\theta_{\text {jet }}}{0.1}\right)\left(\frac{\varepsilon_{\gamma}}{\mathrm{TeV}}\right)^{2} \mathrm{G} .
$$

To avoid significant deflection in the Mpc scale structured region of galaxy clusters with $\sim 10^{-9} \mathrm{G}$ field (Ryu et al. 2008; Murase et al. 2011), the coherence length scale should be $\lesssim 10 \mathrm{kpc}$ from Equation (9). For much larger magnetic field and/or coherence length the UHECRs become isotropic and their luminosity decreases by a beaming factor $f_{b}=\left(1-\cos \theta_{\text {jet }}\right)^{-1} \approx 200$ for $\theta_{\text {jet }}=0.1$. As a result our lower limits on UHECR power will become stronger in such a case.

Propagation of UHECRs from the TeV blazars nearly along our line-of-sight, in low IGMF environment, brings up naturally the question of their detectability by the UHECR detectors on the Earth. While the highestenergy CRs are absorbed during propagation in the CMB, since the blazars are beyond the GZK radius, sufficiently energetic CRs can still reach us (see Fig. 1) and we discuss the prospect of their detection in the next section.

\section{DETECTABILITY OF UHECRS FROM TEV BLAZARS}

The location of the Pierre Auger Observatory (Abraham et al. 2004) at latitude $35.2^{\circ} \mathrm{S}$ makes it insensitive to the nearest and most powerful UHECR candidate source 1ES $1426+428$ with a Declination $\delta=+42.67^{\circ}$. The proposed Auger North detector at latitude $39^{\circ} \mathrm{N}$, however, will be sensitive to $1 \mathrm{ES}$ $1426+428$. For a detector similar to the Auger South, the instrument exposure becomes independent of energy at $E \geq 3 \times 10^{18} \mathrm{eV}$ and depends only on the zenith angle $\theta_{z}$ (Abraham et al. 2008). For Auger South $\theta_{z} \leq 60^{\circ}$. The relative exposure at a point source in the sky, such as 1ES 1426+428, compared to the largest exposure on the sky (Sommers 2001) is $\omega(\delta)=0.62$ for the Auger North. The actual exposure on the source can be found from multiplying $\omega(\delta)$ by the total integrated exposure $\mathcal{E}\left(\mathrm{km}^{2}\right.$ yr $\left.\mathrm{sr}\right)$ over the detector's field-of-view and dividing by the solid angle of the detector $\Omega=\sqrt{3} \pi \mathrm{sr}$, for $\theta_{z} \leq 60^{\circ}$ (see also Cuoco \& Hannestad 2008). Thus the total number of UHECR events expected from $1 \mathrm{ES} 1426+428$, for $\kappa=2.2$ source spectrum, in the proposed Auger North detector is

$$
N_{\text {evt }}=\frac{\mathcal{E} \omega(\delta)}{\Omega} \int_{E_{\mathrm{th}}}^{E_{\mathrm{mx}}} J(E) d E
$$

$$
\approx 3\left(\frac{L_{p}}{10^{46} \mathrm{erg} / \mathrm{s}}\right)\left(\frac{\mathcal{E}}{9,000 \mathrm{~km}^{2} \mathrm{yr} \mathrm{sr}}\right),
$$

above a threshold energy $E_{\mathrm{th}}=4 \times 10^{19} \mathrm{eV}$ and below the maximum energy $E_{\mathrm{mx}}=10^{20} \mathrm{eV}$. Thus 1ES 1426+428 should be clearly visible, if an UHECR source as modeled here, with the same exposure used in the first data release by the Auger South Observatory (Abraham et al. 2008).

The arriving UHECRs from 1ES $1426+428$ are expected to be deflected by the Galactic magnetic field (see, e.g., Dermer et al. 2009) by an angle

$$
\begin{aligned}
\theta_{\mathrm{Gal}}= & 1.5^{\circ}\left(\frac{E}{4 \times 10^{19} \mathrm{eV}}\right)^{-1}\left(\frac{B_{\mathrm{Gal}}}{10^{-6} \mathrm{G}}\right) \\
& \times\left(\frac{h_{\mathrm{disc}}}{\mathrm{kpc}}\right)\left(\frac{\sin b}{\sin 64.9^{\circ}}\right)^{-1},
\end{aligned}
$$

where $h_{\text {disc }} \sim 1 \mathrm{kpc}$ is the height of the Galactic disc and $b=64.9^{\circ}$ is the galacic latitude of 1ES $1426+428$. The deflection angle while propagating in the IGMF, with $B_{\mathrm{IGMF}, \mathrm{TeV}} \lesssim 10^{-16} \mathrm{G}$, is negligible. Thus UHECRs from 1ES $1426+428$ should be clustered around the source direction for a Northern site detector similar to Auger South with $\sim 1^{\circ}$ or better resolution above $\sim 10^{19} \mathrm{eV}$ (Abraham et al. 2008).

It is interesting to note that the position of 1ES $1426+428$ is within $\sim 5^{\circ}-10^{\circ}$ of three clustered (triplet) events detected by the AGASA experiment during its operation with energies $4.97 \times 10^{19} \mathrm{eV}$, $4.98 \times 10^{19} \mathrm{eV}$ and $5.27 \times 10^{19} \mathrm{eV}$ (Takeda et al. 1999; Havashida et al. 2000). The average angular resolution of AGASA however is $1.8^{\circ}$, smaller than the angular separation between the triplet and 1ES 1426+428. Downgrading the event energies by $25 \%$ (Takeda et al. $2003)$ does not increase the deflection angle $\theta_{\text {Gal }}$ significantly. If, however, $B_{\text {Gal }} \sim 4 \times 10^{-6} \mathrm{G}$ in the Galactic disc, $\theta_{\mathrm{Gal}}$ can be large enough to encompass the nearest AGASA event from the triplet. Although the exposure is lower compared to its peak exposure in other directions in the sky, HiRes experiment has not detected any event above $4 \times 10^{19} \mathrm{eV}$ from the direction of 1ES 1426+428 (Abbasi et al. 2010).

The detection prospect for UHECRs with $\kappa=2.2$ from the other 3 blazars within the field-of-view of the Auger South is much smaller, $N_{\text {evt }}=0.04,0.03$ and 0.02 respectively for $1 \mathrm{ES} 0229+200\left(\delta=+20.27^{\circ}\right)$, 1ES 1101$232\left(\delta=-23.50^{\circ}\right)$ and 1ES 0347-121 $\left(\delta=-11.98^{\circ}\right)$; for $L_{p}=10^{45} \mathrm{erg} / \mathrm{s}$ and $\mathcal{E}=9,000 \mathrm{~km}^{2}$ yr sr.

\section{DISCUSSION AND CONCLUSIONS}

The lower limits on the UHECR power $L_{\mathrm{UHECR}}$, in the $10^{17}-10^{20} \mathrm{eV}$ range, that we have derived are lower than the synchrotron luminosity $L_{\mathrm{syn}}$ of the sources for $\kappa=2.2$, except for $1 \mathrm{ES} 1426+428$ where $L_{\mathrm{UHECR}} / L_{\mathrm{syn}} \sim$ 6,15 , and 30 for $\kappa=2.2,2.5$, and 2.7 respectively. While none of these sources have been detected by the Fermi LAT (Abdo et al. 2010a), $L_{\text {UHECR }}$ is a factor $\sim 1-60$ times the median $\gamma$-ray luminosity of $\sim 3 \times 10^{45} \mathrm{erg} / \mathrm{s}$, in the LAT range, from the $118 \mathrm{BL}$ Lacs detected by Fermi with known redshift (Abdo et al. 2010a). The ratio $L_{\text {UHECR }} / L_{\text {syn }}$ gets smaller in the case TeV blazars generate cosmic rays in the $10^{18}-10^{20} \mathrm{eV}$ range only. 
The lower limits on the jet power that we have calculated, exceed $L_{\text {syn }}$ and the median BL Lac $\gamma$-ray luminosity in the Fermi LAT range for all the blazars we considered. In the most extreme case of $1 \mathrm{ES} 1426+428$ the ratio $L_{\text {jet }} / L_{\text {syn }} \sim 2 \times 10^{2}, 5 \times 10^{4}, 2 \times 10^{6}$ for the single power-law spectrum with $\kappa=2.2,2.5,2.7$ respectively. Even for $\kappa=2.2$, the limiting Eddington luminosity is violated in this case by an order of magnitude. For other blazars, $L_{\text {jet }} \lesssim L_{\text {Edd }}$ for $\kappa=2.2$ and single power-law spectrum. However $L_{\text {jet }} \gtrsim L_{\mathrm{Edd}}$ for $\kappa>2.2$, both for the single power-law and broken power-law spectra.

If the observed $\mathrm{TeV} \gamma$ rays from the blazars that we have considered are due to UHECR acceleration in these sources and due to their energy losses while propagating along the line-of-sight, the source spectrum of UHECRs needs to be $\propto E^{-2.2}$ or harder in order for the model to be energetically viable. If the synchrotron luminosity is a good indicator of the contribution by the relativistic electrons to the jet power, then the power in baryons with Lorentz factor $\gamma_{p} \geq 1$ in the relativistic jet needs to be larger by a factor of 200 or more in the case of $1 \mathrm{ES} 1426+428$ with $\kappa=2.2$. The actual jet power will be even higher when including non-relativistic baryons in the jet. The proposed Auger North Observatory should be able to detect CRs above $4 \times 10^{19} \mathrm{eV}$ from $1 \mathrm{ES} 1426+428$ within a few years of operation and test the model of $\mathrm{TeV} \gamma$ ray origin from UHECRs as proposed by Essey et al. (2010, 2011) and as discussed here. Detection of GZK neutrinos from 1ES $1426+428$ by the currently operating IceCube neutrino observatory at the South Pole and by its proposed extensions could be possible. Non-detection of UHECRs from 1ES $1426+428$ can imply a large IGMF as generally inferred from a lack of significant correlation between the sources and UHECR arrival directions, and/or favor leptonic production mechanism of $\mathrm{TeV} \gamma$ rays.

To fit the observed UHECR spectrum as measured by the Auger and HiRes arrays, with protons, an injection spectrum $\propto E^{-2.7}$ above $10^{18} \mathrm{eV}$ is required to explain the dip and cutoff as due to $e^{+} e^{-}$pair production and $\pi$ production, respectively (Berezinsky 2008). A broken power law $\propto E^{-2.7}$ above and $\propto E^{-2.2}$ below a break-energy $\sim 10^{17}-10^{18} \mathrm{eV}$ can help reduce the energy crisis in the AGN interpretation of the observed UHECRs. This scenario also makes the energy requirement for the UHECR interpretation of TeV $\gamma$ rays from blazars less severe. To match the observed spectrum, the total energy injection rate in UHECRs above $10^{17} \mathrm{eV}$ in the local universe needs to be $\sim 10^{45} \mathrm{erg} \mathrm{Mpc}^{-3} \mathrm{yr}^{-1}$ (see e.g. Vietri et al. 2003; Berezinsky et al. 2006). The energy injection rates above $10^{17} \mathrm{eV}$ from the $4 \mathrm{TeV}$ blazars that we considered are $(\gtrsim 2, \gtrsim 6, \gtrsim 11) \times 10^{45} f_{b}^{-1}$ erg $\mathrm{Mpc}^{-3} \mathrm{yr}^{-1}$, respectively, for $\kappa=(-2.2,-2.5,-2.7)$ from our lower limits on UHECR power. The energy injection rates are calculated from the UHECR powers divided by the comoving volumes corresponding to the redshifts of each blazar (Dermer \& Razzaque 2010). If the structured region with high magnetic field near a $\mathrm{TeV}$ blazar makes $\gtrsim 10^{17} \mathrm{eV}$ cosmic rays isotropic, our lower limits on UHECR power will be higher by a factor $\sim f_{b}$ and the corresponding energy injection rate could be sufficient to explain the observed UHECR spectrum above $\sim 10^{17} \mathrm{eV}$. There will be many $\mathrm{TeV} \gamma$-ray sources without the $\mathrm{UV} / \mathrm{X}$-ray synchrotron counterparts from the misaligned blazars in this scenario, which could be searched for with existing and future telescopes.

The jet power exceeds the Eddington luminosity in almost all cases we considered and will do so more severely if the UHECRs become isotropic in the scenario discussed above. It is possible that extragalactic sources with hard injection spectrum contribute to the observed UHECRs far above $\sim 10^{17} \mathrm{eV}$, as explored in the GRB model with $\kappa \sim 2.2$ above the ankle at $\sim 4 \times 10^{18} \mathrm{eV}$ and with observed star-formation rate (Dermer \& Atovan 2006). However, in such models an additional component of UHECRs in the intermediate $\sim 10^{17}-4 \times 10^{18} \mathrm{eV}$ energy range is required. In principle they can originate from low-luminosity GRBs/hypernovae of either extragalactic (Wang et al. 2007) or Galactic (Wick et al. 2004; Budnik et al. 2008; Calvez et al. 2010) origin. Whether nature conspires to generate such a high-energy break in the injection spectrum or the observed UHECRs originate in multiple source classes is a matter of ongoing investigations. However, the interpretation of $\mathrm{TeV} \gamma$ rays from blazars generating UHECRs seems less favorable in case the injection spectrum is similar to the one required to explain the observed cosmic-ray spectrum above $\sim 10^{17}-10^{18} \mathrm{eV}$.

\section{ACKNOWLEDGMENTS}

We thank M. Böttcher, C. C. Cheung, L. Costamante, M. Mostafa and K. Murase for helpful comments and discussion. We also thank the anonymous referee for a constructive report. This work is supported by grants from the NASA Fermi Cycle 3 guest investigator program and by the Office of Naval Research. Work of S.R. was funded while under contract with the U.S. Naval Research Laboratory.

\section{REFERENCES}

Abbasi, R. U., et al. 2010, ApJ, 713, L64

Abdo, A. A., et al. 2010a, ApJ, 715429

Abdo, A. A., Ackermann, M., Ajello, M., et al. 2010b, ApJS, 188, 405

Abdo, A. A., et al. 2010c, ApJ, 723, 1082

Abraham, J., et al. 2004, Nuclear Instruments and Methods in Physics Research A, 523, 50

Abraham, J., et al. 2008, Astroparticle Physics, 29, 188

Abraham, J. et al. 2009, arXiv:0906.2347

Aharonian, F., et al. 2003, A\&A, 403, 523

Aharonian, F., et al. 2007a, A\&A, 470, 475

Aharonian, F., et al. 2007b, A\&A, 473, L25

Aharonian, F., et al. 2007c, A\&A, 475, L9
Aharonian, F., Buckley, J., Kifune, T., \& Sinnis, G. 2008, Reports on Progress in Physics, 71, 096901

Band, D., et al. 1993, ApJ, 413, 281

Barrow, J. D., Ferreira, P. G., \& Silk, J. 1997, Phys. Rev. Lett., 78,3610

Berezinsky, V., Gazizov, A. Z., \& Grigorieva, S. I. 2005, Physics Letters B, 612, 147

Berezinsky, V., Gazizov, A., \& Grigorieva, S. 2006, Phys. Rev. D, 74,043005

Berezinsky, V. 2008, Advances in Space Research, 41, 2071

Böttcher, M. 2007, Ap\&SS, 309, 95

Budnik, R., Katz, B., MacFadyen, A., \& Waxman, E. 2008, ApJ, 673, 928 
Calvez, A., Kusenko, A., \& Nagataki, S. 2010, Phys. Rev. Lett., 105, 091101

Costamante, L., \& Ghisellini, G. 2002, A\&A, 384, 56

Cuoco, A., \& Hannestad, S. 2008, Phys. Rev. D, 78, 023007

Dai, Z. G., Zhang, B., Gou, L. J., Mészáros, P., \& Waxman, E. 2002, ApJ, 580, L7

Dermer, C. D., \& Atoyan, A. 2006, New Journal of Physics, 8, 122

Dermer, C. D., Razzaque, S., Finke, J. D., \& Atoyan, A. 2009, New J. Phys. 11, 065016

Dermer, C. D., \& Menon, G. 2009, High Energy Radiation from Black Holes (Princeton, NJ: Princeton Univ. Press)

Dermer, C. D., \& Razzaque, S. 2010, ApJ, 724, 1366

Dermer, C. D., Cavadini, M., Razzaque, S., Finke, J. D., Chiang, J., \& Lott, B. 2011, ApJ, 733, L21

Dolag, K., Kachelriess, M., Ostapchenko, S., \& Tomàs, R. 2011, ApJ, 727, L4

Essey, W., \& Kusenko, A. 2010, Astroparticle Physics, 33, 81

Essey, W., Kalashev, O., Kusenko, A., \& Beacom, J. F. 2010, Phys. Rev. Lett., 104, 141102

Essey, W., Kalashev, O., Kusenko, A., \& Beacom, J. F. 2011, ApJ, 731, 51

Fazio, G. G., \& Stecker, F. W. 1970, Nature, 226, 135

Finke, J.D., Razzaque, S., \& Dermer, C.D. 2010, ApJ, 712, 238

Franceschini, A., Rodighiero, G., \& Vaccari, M. 2008, A\&A, 487, 837

Gaisser, T. K. 2010, arXiv:1010.5996

Giommi, P., \& Padovani, P. 1994, MNRAS, 268, L51

Greisen, K. 1966, Phys. Rev. Lett., 16, 748

Hampf, D., Tluczykont, M., \& Horns, D. 2011, arXiv:1104.2336

Hayashida, N., et al. 2000, arXiv:astro-ph/0008102

Hogg, D. W. 1999, arXiv:astro-ph/9905116

Ichiki, K., Inoue, S., \& Takahashi, K. 2008, ApJ, 682, 127

Kahniashvili, T., Maravin, Y., \& Kosowsky, A. 2009, Phys. Rev. D, 80, 023009

Mücke, A., Protheroe, R. J., Engel, R., Rachen, J. P., \& Stanev, T. 2003, Astroparticle Physics, 18, 593
Murase, K., Ioka, K., Nagataki, S., \& Nakamura, T. 2006, ApJ, 651, L5

Murase, K., Dermer, C. D., Takami, H., \& Migliori, G. submitted to ApJ (arXiv:1107.5576

Neronov, A., \& Vovk, I. 2010, Science, 328, 73

Orr, M. R., Krennrich, F., \& Dwek, E. 2011, ApJ, 733, 77

Perkins, J. S. [VERITAS Collaboration 2010], BAAS, 42, 708

Pe'er, A., Murase, K., \& Mészáros, P. 2009, Phys. Rev. D, 80, 123018

Ptuskin, V., Zirakashvili, V., \& Seo, E.-S. 2010, ApJ, 718, 31

Rachen, J. P., \& Biermann, P. L. 1993, A\&A, 272, 161

Razzaque, S., Mészáros, P., \& Zhang, B. 2004, ApJ, 613, 1072

Razzaque, S., Dermer, C. D., \& Finke, J. D. 2010, Open Astron. J. 3, 150

Ryu, D., Kang, H., Cho, J., \& Das, S. 2008, Science, 320, 909

Sommers, P. 2001, Astropart. Phys., 14, 271

Stanev, T., Engel, R., Mücke, A., Protheroe, R. J., \& Rachen, J. P. 2000, Phys. Rev. D, 62, 093005

Stecker, F. W., Malkan, M. A., \& Scully, S. T. 2006, ApJ, 648, 774

Takeda, M., et al. 1999, ApJ, 522, 225

Takeda, M., et al. 2003, Astroparticle Physics, 19, 447

Tavecchio, F., Ghisellini, G., Ghirlanda, G., Foschini, L., \& Maraschi, L. 2010a, MNRAS, 401, 1570

Tavecchio, F., Ghisellini, G., Foschini, L., Bonnoli, G., Ghirlanda, G., \& Coppi, P. 2010b, MNRAS, 406, L70

Taylor, A. M., Vovk, I., \& Neronov, A. 2011, A\&A, 529, A144

Vietri, M. 1995, ApJ, 453, 883

Vietri, M., De Marco, D., \& Guetta, D. 2003, ApJ, 592, 378

Wang, X.-Y., Razzaque, S., Mészáros, P., \& Dai, Z.-G. 2007,

Phys. Rev. D, 76, 083009

Waxman, E. 1995, Phys. Rev. Lett., 75, 386

Wick, S. D., Dermer, C. D., \& Atoyan, A. 2004, Astropart. Phys. 21,125

Zatsepin, G. T., \& Kuz'min, V. A. 1966, Soviet Journal of

Experimental and Theoretical Physics Letters, 4, 78 